\title{
Caractérisation morphologique de Cleome gynandra L. au Bénin
}

\author{
Yédjanlognon Faustin ASSONGBA ${ }^{1,3^{*}}$, Jean Innocent ESSOU ${ }^{2}$, Cossi Aristide ADOMOU ${ }^{2}$ et \\ M.G. Julien DJEGO ${ }^{3}$ \\ ${ }^{1}$ Ecole Nationale Supérieure des Biosciences et Biotechnologies Appliquée (ENSBBA), Université Nationale, des Sciences, \\ Technologies, Ingénierie et Mathématiques (UNSTIM) Abomey \\ ${ }^{2}$ Département Biologie Végétale, FAST/UAC, 01BP4521 Cotonou/Bénin \\ ${ }^{3}$ Laboratoire d'Ecologie Appliquée (LEA) / FSA / UAC \\ *Auteur correspondant ; E-mail: yedjanlognon@gmail.com/yedjanlognon@yahoo.fr
}

Received: 27-06-2017

Accepted: 04-12-2017

Published: 28-02-2021

\section{RESUME}

Au Bénin des efforts scientifiques sont consentis pour la connaissance de la diversité végétale. Cleome gynandra L. (Caya blanc, Sabo en Adja, Akaya en Mahi et Semboué en Peulh) de la famille des Capparaceaes fait l'objet de cette étude à travers sa connaissance agromorphologique. La méthodologie utilisée passe par des observations et prélèvement dans la nature et jardins de case. Dix (10) pieds de la plante ont été collectés dans chaque zone climatique et un (1) pied dans le jardin botanique de l'Université d'Abomey-Calavi. En plus des observations sur la plante complète, il est procédé à l'achat des graines de $C$. gynandra. Des variables quantitatives (hauteur de la plante, nombre de fruits par pied, longueur et diamètre des fruits, celle des pédoncules, nombre de ramification) et variables qualitatives (couleur, pilosité de la tige, couleur des fruits et graines et forme des feuilles) ont été observées, mesurées et calculées. Le traitement des variables a été possible par usage du logiciel XLSTAT- pro Version 2008. 1.01. Les relations entre variables ont été étudiées grâce à la matrice de corrélation de Pearson (n). Une Analyse en Composante Principale (ACP), a permis de ressortir si des différences significatives à un taux de 0,05 existent entre les variétés pour les caractères étudiés. L'étude a mis en évidence une grande variabilité agromorphologique dans les trois zones climatiques du Bénin par des caractères discriminant. Des différences significatives ont été observées entre les variables des trois zones climatiques d'une part et entre les individus issus des jardins de case et ceux collectés dans la nature d'autre part. L'ACP a mis en évidence trois grands ensembles. La classification ascendante hiérarchisée montre (8) ensembles qui tiennent compte des similarités et les observations.

(C) 2021 International Formulae Group. All rights reserved.

Mots clés : Cleome gynandra L, Akaya, variété, caractères, légume feuille et République du Bénin.

\section{Morphological characterization of Cleome gynandra L. in Benin}

\begin{abstract}
In Benin, scientific efforts are being made for knowledge of plant diversity. Cleome gynandra L. (White Caya, Sabo in Adja, Akaya in Mahi and Semboué in Peulh) of the family Capparaceae is the subject of this study through its agromorphological knowledge. The methodology used involves observations and sampling in the nature and house gardens. Ten (10) feet of the plant were collected in each climatic zone and one (1) foot in the botanical garden of the University of Abomey-Calavi. In addition to the observations on the
\end{abstract}


whole plant, the seeds of $C$. gynandra are purchased. Quantitative variables (plant height, number of fruits per foot, fruit length and diameter, peduncles, number of branching) and qualitative variables (color, stem hair, fruit and seed color and leaf forms) were observed, measured and calculated. Variable processing was possible using the XLSTAT-pro Version 2008 software. 1.01. Relationships between variables were studied using the Pearson correlation matrix (n). A Principal Component Analysis (PCA) revealed if significant differences at a rate of 0.05 exist between the varieties for the characters studied. The study revealed a large agromorphological variability in the three climatic zones of Benin by discriminating characters. Significant differences were observed between the variables of the three climatic zones on the one hand and between the individuals from the house gardens and those collected in the wild on the other hand. The CPA has highlighted three major groups. The hierarchical ascending classification shows (8) sets that take into account similarities and observations.

(C) 2021 International Formulae Group. All rights reserved.

Keywords: Cleome gynandra L, Akaya, variety, characters, leaf vegetable and Republic of Benin.

\section{INTRODUCTION}

Caya blanc ou Moustache de chat (Cleome gynandra L.) (Capparaceae) est une espèce végétale annuelle, pantropicale, largement répandue en Afrique (Mnzava et Ngwerume, 2004 ; Short, 2011 ; Meda et al., 2013 ; Diarrassouba et al., 2014) et consommé comme légume-feuille (Silué, 2009 ; Agbo et al., 2014.). Selon l'OMS (2011) C. gynandra est une plante dont les feuilles sont utilisées pour les problèmes de malnutrition qui est courante dans de nombreux pays. Les estimations récentes montrent que près de 178 millions d'enfants souffrent d'un retard de croissance, un indicateur clé de la malnutrition chronique (OMS, 2011). Ce constat est confirmé par les auteurs MillogoRasolodimby (2001), Hari (2013) et Kièbre et al. (2015) qui ont montré que les feuilles de $C$. gynandra sont utilisées pendant les périodes de soudure. Ainsi, avec les crises alimentaires, la consommation de $C$. gynandra connait une augmentation et attirant un grand nombre de phytothérapeutes. Au Bénin, C. gynandra est entrain de suppléer les légumes «Gboma» (Solanum macrocarpon L.) du fait de sa disponibilité (régénérescence par graines). Le suc de ses feuilles fraîches est utilisée dans le traitement des ictères et hyperthermies (Akoegninou et al., 2006 ; Dansi et al., 2012). $\mathrm{Au}$ Bénin, malgré l'importance socioéconomique, alimentaire et médicinale de l'espèce, très peu d'études sont consacrées sur la caractérisation morphologique de cette plante. Quelques études réalisées au Kenya (K'Opondo et al., 2009 ; K’Opondo, 2011) et au Zimbabwe (Masuka et Mazarura, 2012) ont montré l'existence de diversités au sein de l'espèce, au Bénin, aucune étude spécifique sur la diversité de cette plante n'est encore réalisée. En effet, Cleome gynandra a été négligée suite à l'introduction des légumes exotiques (Abukutsa, 2010). La connaissance de la caractérisation morphologique étant indispensable à la connaissance variétale, il est nécessaire d'entreprendre des études sur la caractérisation morphologique au Bénin de $C$. gynandra car des observations de terrain montrent que la morphologie de la plante varie en fonction de la zone éco-climatique. L'utilisation de paramètres morphologiques permet une bonne appréciation des potentialités adaptatives de $C$. gynandra et serait un outil de la caractérisation variétale et phénotypique. La disponibilité des données sur la caractérisation morphologique de $C$. gynandra permettra d'asseoir un programme de valorisation et de conservation de l'espèce au Bénin. Cette étude est une analyse du polymorphisme de C. gynandra au Bénin.

\section{MATERIEL ET METHODE}

La République du Bénin, pays de l'Afrique de l'Ouest est située entre les parallèles $6^{\circ} 30^{\prime}$ et $12^{\circ} 30^{\prime}$ de latitude Nord et entre les méridiens $1^{\circ}$ et $3^{\circ} 40^{\prime}$ de longitude Est. Elle s'étend sur une superficie totale de $114.763 \mathrm{~km}^{2}$ si l'on considère les îles sur le 
fleuve Niger (Assongba, 2014). Il est constitué de trois zones climatiques à savoir : la zone guinéenne, guinéo-soudanienne et soudanienne et possède plusieurs aires protégées (Figure 1). Le climat est de type subéquatorial au Sud, tropical humide de transition au Centre et tropical sec au Nord du pays (Akoégninou, 2004). Le pays compte 9983884 habitants (INSAE, 2013). Neuf (9) groupes sociolinguistiques y sont distingués : fon et apparentés $(39,2 \%)$, adja et apparentés $(15,2 \%)$, yoruba et apparentés $(14,5 \%)$, bariba et apparentés $(09,2 \%)$, peulh et apparentés $(07,3 \%)$, ottamari et apparentés $(06,4 \%)$, yoaLokpa et apparentés $(04,3 \%)$, dendi et apparentés (02,5\%), autres $(01,6 \%)$ constituées de zerma, haoussa, cotimba (INSAE, 2013; FAOSTAT, 2010). Le Tableau 1 présente les caractéristiques climatiques, coordonnées géographiques des zones investiguées, le type de sol et altitude des milieux investigués.

\section{METHODOLOGIES DE COLLECTE ET TRAITEMENT DES DONNEES \\ Matériel végétal et sites de collecte}

Le matériel végétal est constitué de 31 échantillons à raison dix (10) pieds de Cleome gynandra par zone climatique et un (1) pied dans le jardin botanique de l'Université d'Abomey-Calavi à cause du caractère de conservateur du jardin. Les différentes parties de la plante constituent également le matériel biologique végétal (graine, fleur, fruit).

\section{Description botanique et importance de Cleome gynandra}

Cleome gynandra est une herbacée atteignant environ $60 \mathrm{~cm}$ de hauteur. Ses feuilles sont composées, longuement pétiolées avec 3 à 7 folioles. Les fleurs de la plante présentent une couleur variant de blanc à pourpre avec des pétales s'amincissant brusquement en un long onglet basal. Sa floraison et sa fructification a lieu entre avril et décembre. Les fruits sont des capsules linéaires et cylindriques. Cleome gynandra est une plante alimentaire, médicinale, fourragère et ornementale. Elle n'est plus considérée comme une adventiste, mais comme une comestible et une source de revenue (Masuka et Mazarura, 2012 ; Kiebre et al., 2015). Cleome gynandra est récolté dans la nature ou cultivé dans les jardins de case comme légume-feuille (Silué, 2009; Hari, 2013.). Cette plante s'adapte aux conditions locales que les légumes importés. Au Bénin, la plante est retrouvée dans les jachères, les décombres, jardins de case et bords de route (Bouet et al., 2014). Cleome gynandra est un légume-feuille à fort teneur des vitamines $\mathrm{A}, \mathrm{C}, \beta$-carotène, fer, calcium, magnésium, protéines, phosphore (Soro et al., 2012 ; Kiebre et al., 2015).

\section{COLLECTE DES DONNEES \\ Variables morphologiques collectées}

Six (6) variables quantitatives (la hauteur de la plante, le nombre de fruits par pied, la longueur des fruits, le diamètre des fruits, la longueur des pédoncules, le nombre de ramification) et cinq (5) variables qualitatives (la couleur et pilosité de la tige, la couleur des fruits et des graines, la forme des feuilles) ont été observées, mesurées et calculées. Ce sont des paramètres nécessaires pour la caractérisation morphologique (IPGRI, 2002 et 2003).

\section{Traitement des données}

Les différents calculs (moyenne, écarttype, maximum et minimum) et analyses statistiques ont été faites sur les diverses variables.

Le traitement des paramètres (variables) a été possible grâce au logiciel XLSTAT- pro Version 2008. 1.01. Les relations entre variables ont été étudiées grâce à la matrice de corrélation de Pearson (n). Une Analyse en Composante Principale (ACP) a été réalisée afin de faire ressortir les différentes similarités existantes entre les variables des caractères étudiés.

L'ACP a permis aussi de voir comment se répartissent les individus de l'espèce. Les résultats de l'Analyse en Composante 
Principale et les coordonnés des pieds de l'espèce étudiés ont servi à une Classification ascendante hiérarchique (CAH) avec troncature automatique, et similarité (coefficient de corrélation de Kendall). Cela a permis de regrouper les populations similaires. Le test de corrélation de Pearson a été réalisé pour tester la corrélation entre les coordonnées géographiques (latitudes longitudes) et les éléments de caractérisations morphologiques.

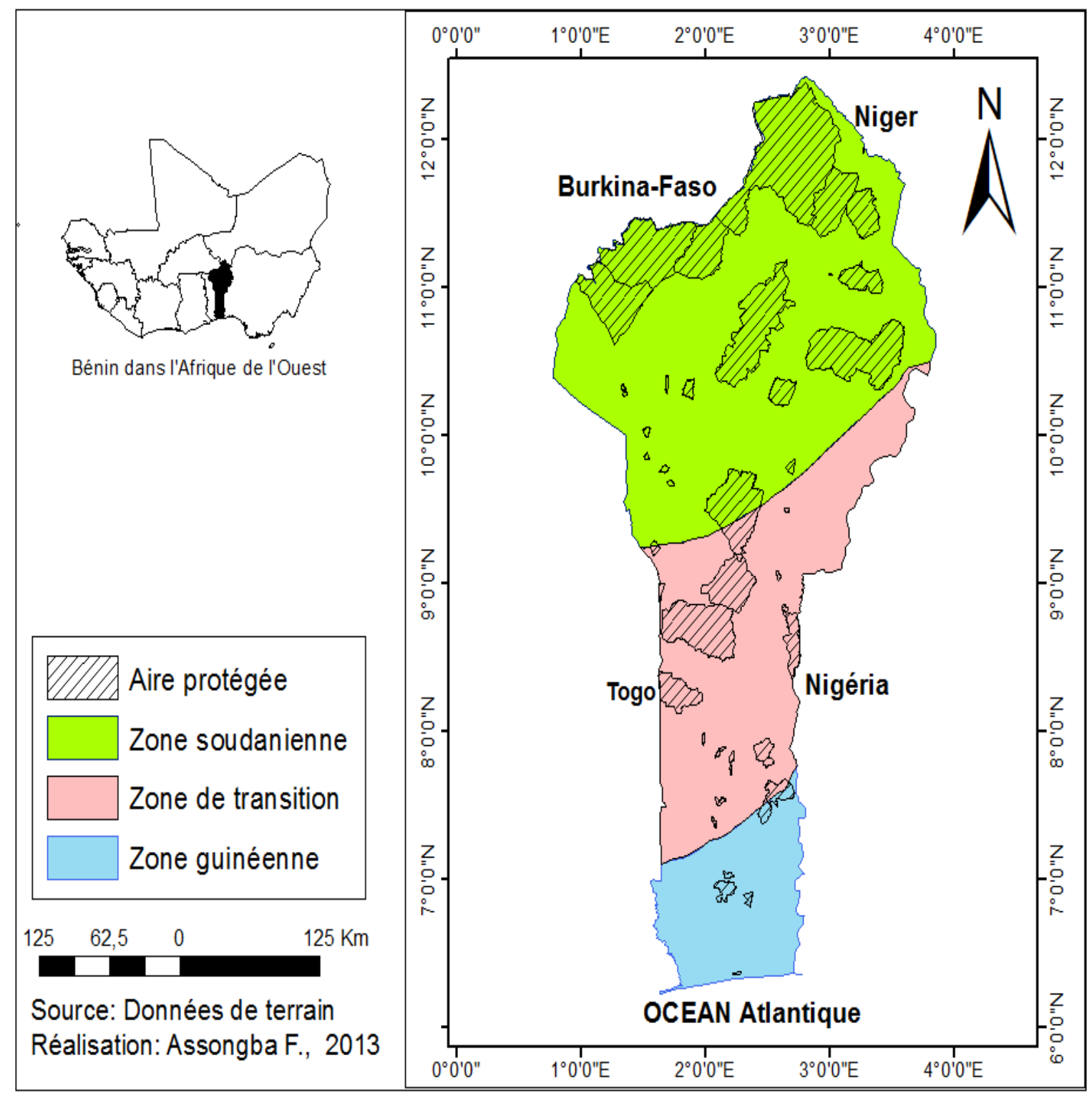

Source : données de terrain

Réalisation, 2017

Figure 1 : Zones climatiques et milieu naturel de l'espèce. 
Tableau 1 : Caractéristiques éco géographiques des zones investigués (Assongba, 2014).

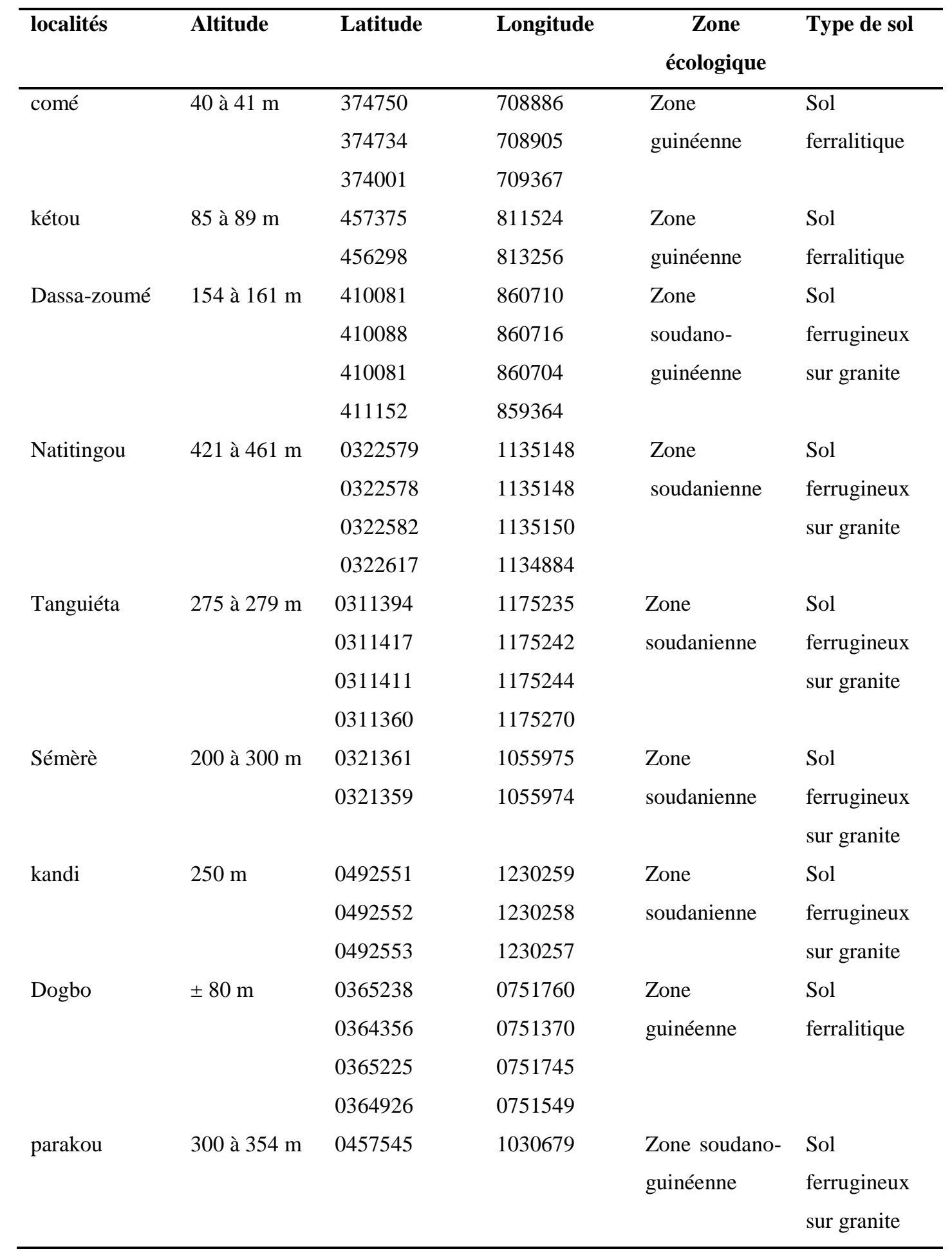




\section{RESULTATS}

\section{Valeur des paramètres quantitatifs}

Le Tableau 2 présente les différentes variables quantitatives par individu de l'espèce. Au total trente un (31) pieds de l'espèce ont servi pour la quantification des paramètres (hauteur, diamètre moyen des fruits, longueur moyenne de pédoncules, la ramification, nombre de fruit par pied.

\section{Structuration de la diversité à partir des variables quantitatives}

L'Analyse en Composante Principale des variables quantitatives indiquent que les deux premiers axes expliquent respectivement $63,73 \%, 16,03 \%$ de la variabilité soit un total de $79,77 \%$ de la variabilité (Tableau 3). L'Analyse en Composante Principale a distingué trois clusters des trois zones climatiques (Figure 2): zones guinéocongolaise, guinéo-soudanienne et soudanienne. La zone guinéo-congolaise regroupe les variétés de Cleome gynandra à port vertical qui peuvent être à tiges rougeverte ou à tiges verte. Les individus de l'espèce qui sont dans cette zone sont à feuilles vertes. Cette zone correspond au climat subéquatorial à deux saisons de pluies où les plantes bénéficient d'une quantité de pluie élevée tout au long de l'année. La zone guinéo-soudanienne regroupe les variétés de Cleome gynandra à tiges verte, couleur des fruits jaune-grise, vert-jaune. C'est la zone de transition qui correspond à un climat de dont les deux pics qui montrent la distinction des deux saisons semblent se coïncider en un seul pic aujourd'hui. Signalons que c'est dans cette zone climatique que les tiges rouges pures ont été observées et qui ne sont pas prise en compte par le regroupement de l'ACP. Enfin le troisième groupe de la zone soudanienne (Figure 2) qui regroupe en son sein des variétés de Cleome gynandra à port horizontal, des tiges de couleur vert-rouge et des feuilles verte-jaune. Cette zone est associée au climat soudanien procédant une seule saison de pluie et une seule saison sèche.
Le Tableau 4 donne les valeurs propres des différents facteurs mis en jeux. Le Tableau 5 regroupe la Corrélation entre les variables et les facteurs. Le Tableau 6 est celui de la matrice Corrélation de Pearson (Corrélation entre milieu et les caractères morphologiques).

\section{Diversité morphologique de Cleome gynandra}

Le dendrogramme (Figure 3) issu de la combinaison des variables qualitatives présente les 31 accessions en 31 observations et 8 classes.

La classe 1 est constituée des observations (obs 1, 2, 6, 7, 8,14, 15, 17, 18, 20, $2122,23,25,27$ et 30), caractérisée par des individus qui ont des diamètres moyens des fruits (DMF) et longueurs moyens des pédoncules (LMPd), presque identiques et un nombre de ramification qui varie entre 3 et 6 . Ces individus sont souvent des pieds de plante ayant les deux couleurs (rouge- verte, ou verte-rouge) et qu'on rencontre dans les trois zones climatiques du Bénin. La classe 2 constituée des observations (obs 3, 4, 5, 12, $13,19,28$, et 31 ), est caractérisée par des pieds de la plante qui ont des longueurs moyennes de fruit (LMF) et longueur moyenne de pédoncule (LMPd) importantes et aussi un nombre élevé de fruits par pied de l'espèce. Les individus de la classe 2 sont de couleur rouge et localisés dans la zone guinéosoudanienne. La couleur rouge ici est pure dans cette zone. La classe 3 (obs9, 11) caractérisent les pieds (individus) qui ont des fruits importants et ayant aussi plus de 5 ramifications. La classe 4 (obs 10) est caractérisée par une importante ramification (8), ce qui donne beaucoup de feuilles et de fruits. Par déduction, les individus de la classe 4 (obs 10) rentrent précocement en production. Ils doivent faire l'objet d'une sélection. La classe 5 (obs 16), possède des individus à ramification petite, des fruits de petite longueur. La classe 6 (obs 24) regroupe des pieds caractérisés par des individus qui semblent ne pas pouvoir porter des fruits. $\mathrm{La}$ 
classe 7 (obs 26) rassemble les pieds caractérisés par une importante ramification (8) ; de long diamètre moyen de fruit. Ceci va entrainer d'importants fruits et par conséquent un taux élevé de germination. La classe 8 (obs 29) regroupe les individus possédant Six (6) ramifications, des ports horizontaux et des fruits importants avec de longueurs moyennes.

\section{Sélection de quelques variétés importantes}

La sélection des graines de ces plantes peut servir de base pour une production à l'échelle industrielle. La sélection naturelle s'observe déjà sur le terrain quand on constate que les pieds ayant les deux couleurs, verterouge ou rouge-verte seraient issue des croisements des lignées pures vertes et de rouges. La recherche d'abondante feuilles pour satisfaire la consommation conduit au choix des variétés qui ont des ramifications importantes et la longueur moyenne des pédoncules importantes car plus le pédoncule est long plus la feuille est large. Or ces caractéristiques correspondent aux observations suivantes et prioritaires (obs 12 et 13), (obs 26, 29,10, 11 et 31) et (obs 5, 6, $17,18$ et 20$)$.

\section{Variabilités observées}

$\mathrm{Au}$ Bénin, trois (3) groupes sont observés en tenant compte de la couleur de la tige. Une tige à couleur verte qui ne varie pas et qui est observée dans les trois zones climatiques du pays. Une tige de couleur rouge pure qui est plus observée au centre du pays et une troisième tige de couleur qui varie beaucoup (verte-rouge, rouge-verte et rouge à la base de la tige et verte au sommet). La tige de couleur rouge a un port horizontal tandis que celle de couleur verte a un port vertical. La couleur verte est plus privilégiée dans les jardins de case et plus utilisée par les populations pour guérir les pathologies. Une variation de la taille de l'espèce est aussi observée. Les individus à grande taille possèdent des fruits et pédoncules longs, un important diamètre. On observe des populations de taille courte et de petits fruits ayant de petit pédoncule. D'autres sont de taille moyenne, fruit et pédoncules moyens. La Figure 4 indique les différents pieds de Cleome gynandra observés au Bénin et les variations de la couleur de la tige.

Tableau 2 : Caractéristiques /paramètres quantitatives des échantillons collectés.

\begin{tabular}{lllllll}
\hline plant & Hp & DMF & LMF & LMPd & Rami & NFrt/Pied \\
\hline Pied1 & 0,45 & 0,29 & 4,04 & 4,21 & 3 & 15 \\
Pied2 & 0,99 & 0,45 & 5,73 & 8 & 4 & 29 \\
Pied3 & 1,1 & 0,41 & 6,94 & 6,22 & 4 & 78 \\
Pied4 & 0,97 & 0,42 & 6,79 & 6,51 & 4 & 116 \\
Pied5 & 0,89 & 0,42 & 7,81 & 6,58 & 3 & 39 \\
Pied6 & 1,8 & 0,54 & 8,69 & 9,06 & 4 & 316 \\
Pied7 & 0,95 & 0,46 & 7,46 & 8,55 & 3 & 40 \\
Pied8 & 0,9 & 0,34 & 5,15 & 8,83 & 3 & 39 \\
Pied9 & 0,75 & 0,3 & 4,79 & 5,62 & 5 & 209 \\
Pied10 & 0,65 & 0,26 & 4,05 & 5,04 & 8 & 192 \\
Pied11 & 0,9 & 0,31 & 5,64 & 6,83 & 6 & 320 \\
\hline
\end{tabular}




\begin{tabular}{lllllll}
\hline Pied12 & 1,12 & 0,45 & 8,24 & 7,69 & 7 & 112 \\
Pied13 & 1,1 & 0,6 & 8,65 & 8,3 & 6 & 151 \\
Pied14 & 0,5 & 0,22 & 3,88 & 4,28 & 2 & 7 \\
Pied15 & 0,55 & 0,25 & 3,42 & 3,7 & 3 & 5 \\
Pied16 & 0,5 & 0,166 & 3,53 & 3,6 & 2 & 3 \\
Pied17 & 1,2 & 0,68 & 6,67 & 9,52 & 5 & 300 \\
Pied18 & 0,97 & 0,55 & 9,3 & 10,53 & 5 & 350 \\
Pied19 & 1,3 & 0,59 & 9,6 & 9,04 & 3 & 283 \\
Pied20 & 0,98 & 0,57 & 7,84 & 9,52 & 5 & 380 \\
Pied21 & 0,95 & 0,45 & 7,27 & 9,13 & 3 & 362 \\
Pied22 & 0,45 & 0,433 & 4,15 & 5,03 & 4 & 10 \\
Pied23 & 0,5 & 0,285 & 3,6 & 4,18 & 3 & 24 \\
Pied24 & 0,55 & 0,35 & 6,175 & 4,025 & 3 & 6 \\
Pied25 & 0,6 & 0,42 & 4,54 & 6,42 & 4 & 22 \\
Pied26 & 0,9 & 0,39 & 7,26 & 6,98 & 8 & 72 \\
Pied27 & 0,65 & 0,44 & 5,17 & 5,46 & 1 & 25 \\
Pied28 & 0,85 & 0,4 & 6 & 5,55 & 1 & 29 \\
Pied29 & 0,7 & 0,33 & 7,27 & 5,09 & 6 & 85 \\
Pied30 & 1,2 & 0,4 & 4,2 & 6,23 & 2 & 32 \\
Pied31 & 1,6 & 0,409 & 6,47 & 6,26 & 6 & 251 \\
\hline (H) & & & 25 & \\
\hline
\end{tabular}

$(\mathrm{Hp}=$ hauteur de la plante, NFrt/pied = Nombre de fruit par pied $; \mathrm{LMF}=$ Longueur Moyenne des Fruits ; DMF = Diamètre Moyen des Fruits ; LMPd = Longueur Moyenne des Pédoncules ; Rami = Ramification).

Tableau 3 : Matrice de corrélation de corrélation Pearson (n).

\begin{tabular}{lllllll}
\hline Variables & Hauteur & DMF & LMF & LMPd & Rami & NFrt/Pied \\
\hline Hauteur & 1 & 0,642 & 0,671 & 0,675 & 0,243 & 0,583 \\
DMF & 0,642 & 1 & 0,759 & 0,820 & 0,155 & 0,541 \\
LMF & 0,671 & 0,759 & 1 & 0,761 & 0,310 & 0,573 \\
LMPd & 0,675 & 0,820 & 0,761 & 1 & 0,244 & 0,685 \\
Rami & 0,243 & 0,155 & 0,310 & 0,244 & 1 & 0,415 \\
& & & & & & \\
NFrt/Pied & 0,583 & 0,541 & 0,573 & 0,685 & 0,415 & 1 \\
\hline
\end{tabular}

Les valeurs en gras sont significativement différentes de 0 à un niveau de signification alpha $=0,05$

$\mathrm{NB}: \mathrm{DMF}=$ diamètre moyen des fruits $; \mathrm{LMF}=$ longueur moyenne des fruits $; \mathrm{LMPd}=$ Longueur moyenne des pédoncules ;

Rami= Ramification ; NFrt= nombre de fruit. 


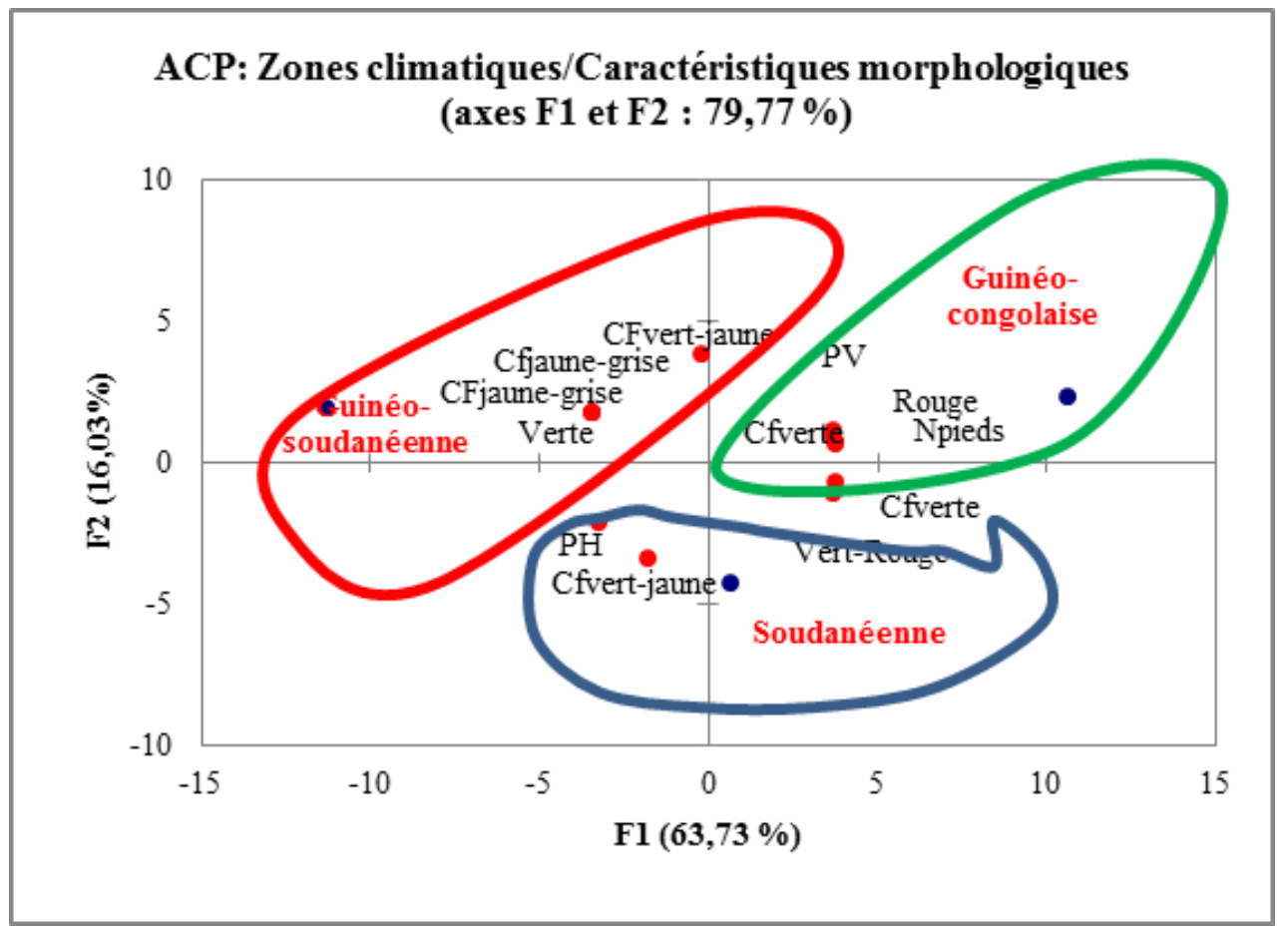

Figure 2 : Projection des 31 individus dans un plan factoriel.

Tableau 4 : Valeurs propres.

\begin{tabular}{lllllll}
\hline & F1 & F2 & F3 & F4 & F5 & F6 \\
\hline Hauteur & 0,420 & $-0,103$ & 0,141 & 0,876 & $-0,157$ & 0,004 \\
DMF & 0,441 & $-0,301$ & $-0,296$ & $-0,278$ & $-0,452$ & $-0,588$ \\
LMF & 0,449 & $-0,089$ & $-0,422$ & $-0,018$ & 0,782 & 0,004 \\
LMPd & 0,466 & $-0,157$ & 0,036 & $-0,301$ & $-0,277$ & 0,767 \\
Rami & 0,210 & 0,896 & $-0,335$ & 0,023 & $-0,199$ & 0,009 \\
NFrt/Pied & 0,406 & 0,250 & 0,775 & $-0,252$ & 0,209 & $-0,255$ \\
\hline
\end{tabular}

$\mathrm{F}=$ facteurs

Tableau 5 : Corrélation entre les facteurs.

\begin{tabular}{lllllll}
\hline & F1 & F2 & F3 & F4 & F5 & F6 \\
\hline Hauteur & 0,822 & $-0,101$ & 0,093 & 0,547 & $-0,076$ & 0,002 \\
DMF & 0,862 & $-0,295$ & $-0,196$ & $-0,173$ & $-0,217$ & $-0,231$ \\
LMF & 0,879 & $-0,087$ & $-0,280$ & $-0,011$ & 0,376 & 0,001 \\
LMPd & 0,912 & $-0,154$ & 0,024 & $-0,188$ & $-0,133$ & 0,301 \\
Rami & 0,410 & 0,879 & $-0,222$ & 0,014 & $-0,096$ & 0,004 \\
NFrt/Pied & 0,795 & 0,245 & 0,513 & $-0,157$ & 0,100 & $-0,100$ \\
\hline
\end{tabular}


Tableau 6 : Corrélation entre milieu et les caractères morphologiques.

\begin{tabular}{lrrrrrrrr}
\hline \multicolumn{1}{c}{ Variables } & La & \multicolumn{1}{l}{ Lon } & \multicolumn{1}{c}{ H } & ram & Nbfrt/Pied & LFrt & diafrt & \multicolumn{1}{l}{ lgPéd } \\
\hline La & 1 & $-0,754$ & 0,408 & 0,288 & 0,178 & 0,238 & 0,181 & $-0,226$ \\
Lon & $-0,754$ & 1 & $-0,548$ & $-0,300$ & $-0,359$ & $-0,39$ & $-0,399$ & $-0,006$ \\
H & 0,408 & $-0,548$ & 1 & 0,765 & 0,729 & 0,701 & 0,891 & 0,408 \\
ram & 0,288 & $-0,300$ & 0,765 & 1 & 0,492 & 0,370 & 0,667 & 0,170 \\
Nbfrt/Pied & 0,178 & $-0,359$ & 0,729 & 0,492 & 1 & 0,847 & 0,836 & 0,657 \\
Lfrt & 0,238 & $-0,398$ & 0,701 & 0,370 & 0,847 & 1 & 0,755 & 0,782 \\
& & & & & & & & \\
Diafrt & 0,181 & $-0,399$ & 0,891 & 0,667 & 0,836 & 0,755 & 1 & 0,460 \\
LgPééd & $-0,226$ & $-0,006$ & 0,408 & 0,170 & 0,657 & 0,782 & 0,460 & 1 \\
\hline
\end{tabular}

Les valeurs en gras sont significativement différentes de 0 à un niveau de signification alpha $=0,05$

$\mathrm{La}=$ latitude $;$ Lon = Longitude $; \mathrm{H}=$ Hauteur Ram = ramification $; \mathrm{LFrt}=$ longueur fruit $; \mathrm{Nbfrt}=$ Nombre de fruit par pied $;$ Diafrt $=$ diamètre fruit $;$ LgPéd $=$ Longueur Pédoncule.

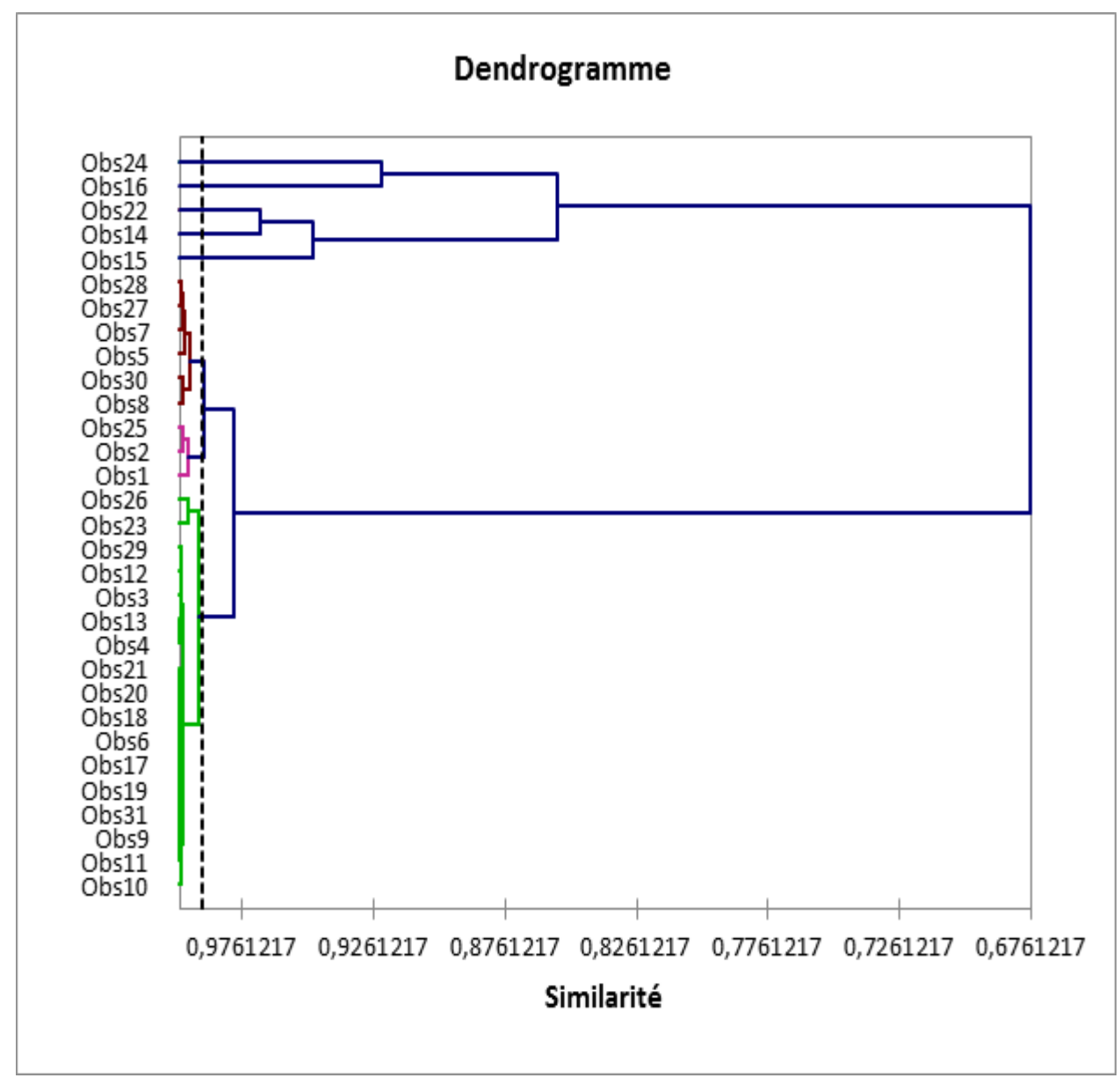

Figure 3 : Classification ascendante hiérarchisée des différentes observations morphologiques de Cleome gynandra. 


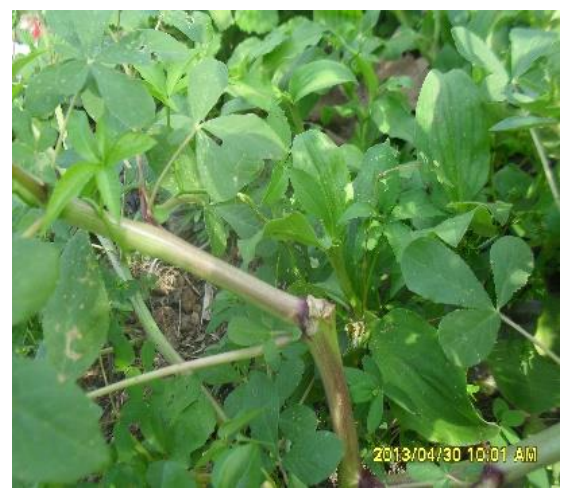

Tige verte
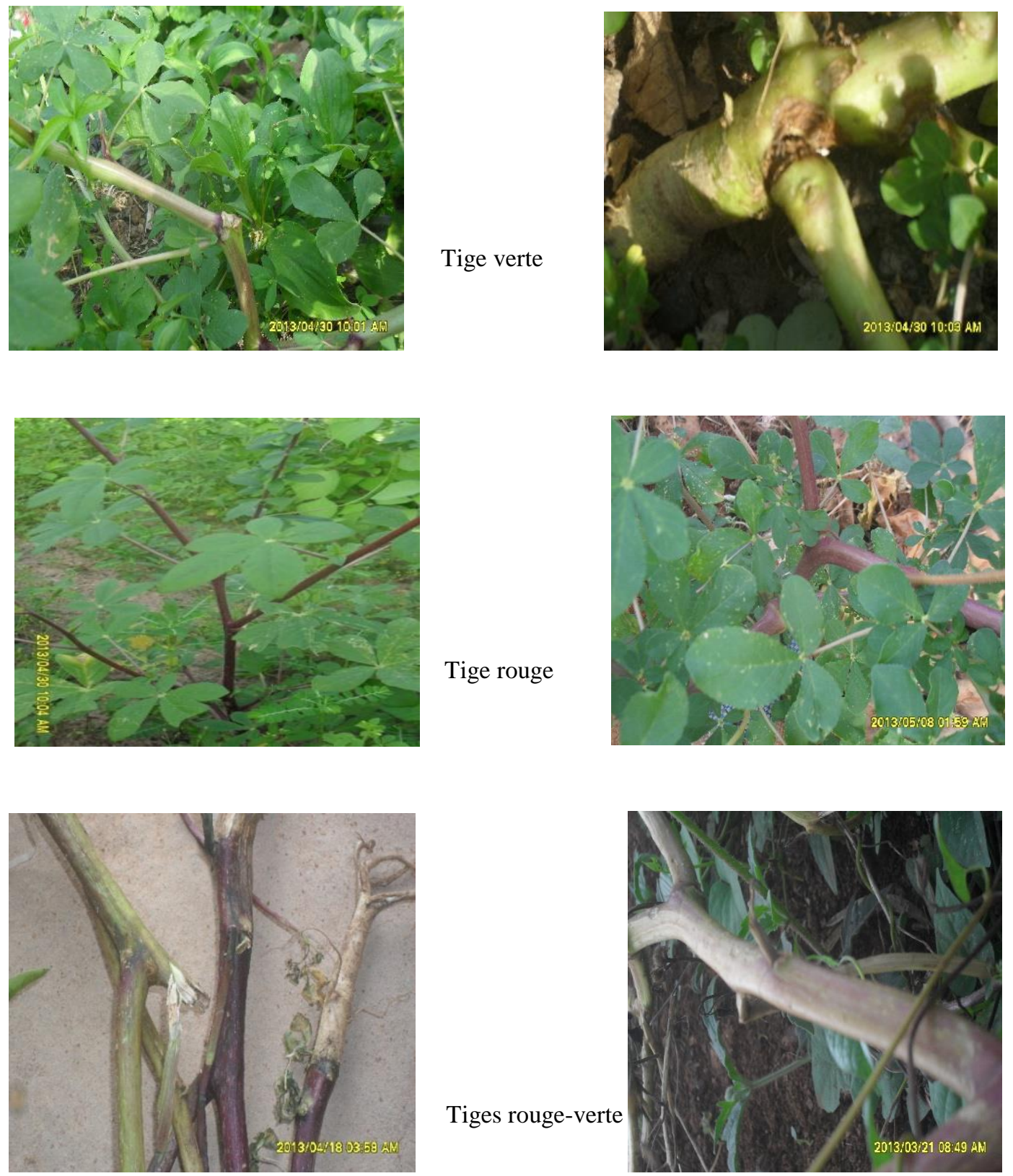

Figure 5 : Les différents pieds de Cleome gyandra observés au Bénin avec la variation de la couleur de la tige.

\section{DISCUSSION}

\section{Variabilité morphologique}

Cette étude a révélé l'existence des variabilités morphologique aux seins des accessions de Cleome gynandra au Bénin. Ce résultat confirme les travaux de K'opondo (2011), de Masuka et al (2012) et de Zakaria et al (2015), effectués sur les morphotypes de
Cleome gynandra respectivement au Kenya, au Zimbabwe et au Burkina-Faso. Selon Agbo et al (2014) C. gynandra est capable d'autofécondation et de fécondation croisée si d'autres individus de C. gynandra sont au voisinage, ceci favorise un brassage génétique naturel conduisant à une diversité génétique importante. Ainsi, la variation des couleurs 
observés surtout les pieds de couleur vertrouge et rouge-verte sont issus des tiges de couleur verte et rouge pure. Quant aux individus de couleur rouge, on note une diversité de la couleur qui est due à la conservation des graines collectées par les paysans qui les vendent plus tard (Zakaria et al., 2015 ; Kiebrè et al., 2016). Cette diversité se rencontre également au niveau de certaines populations de couleur rouge avec une grande ramification (8) et de port horizontale de longueur moyen de fruit (3 à $5 \mathrm{~cm}$ ) au plus, de diamètre moyen entre 0,2 et $0,3 \mathrm{~cm}$ et produisant beaucoup de feuilles et de fruits.

\section{Relations entre les caractères}

La corrélation hautement positive et significative observé entre la hauteur de pied et le diamètre moyen des fruits s'explique par la diversité au sein de Cleome gynandra puisque certaines variétés de cette espèce sont de grandes tailles ce qui explique la longueur et le diamètre de fruit. La longueur des fruits variant entre 5 et $11 \mathrm{~cm}$ et le diamètre des fruits variant entrent 0,4 et $0,6 \mathrm{~cm}$ sont fonction de la hauteur du pied de l'espèce qui donne de longs pédoncules 6 à $12 \mathrm{~cm}$. Ces pédoncules supportant de grandes feuilles favorisent la photosynthèse. Ces grandes feuilles font augmenter la surface foliaire. Ces résultats sont importants pour les sélections des graines et pour une production industrielle. Mais comme dans la nature toutes les choses ne sont jamais égales, on observe des plants nains qui produisent des fruits nains. Ces résultats corroborent les travaux de K'opondo (2011) ; Zakaria et al (2015) et Mariam (2016) sur Cleome gynandra et Corchorus olitorius L. Les relations entre les caractères des végétaux dépendent aussi de l'action de l'homme et des oiseaux pour la prolifération (Bouet et al., 2014).

\section{Organisation de la diversité}

Dans la nature, l'on observe plusieurs variétés $C$. gynandra qui sont ensemble. Mais des critères qualitatifs les distingue c'est le cas de la couleur rouge qui domine la couleur verte. Cette diversité intra s'explique par l'existence d'une grande variété au sein de l'espèce. Ce constat est confirmé par Agbo et al (2014) sur la variation de nutriment contenue dans $C$. gynandra. L'importante de la variabilité des caractères de $C$. gynandra s'explique suivant le gradient climatique due à la pluviométrie, l'isolation et la température élevée dans les zones guinéo-soudanienne et soudanienne. Ces résultats sont confirmés par les travaux de Fandohan et al (2011) sur Tamarindus indica $L$. qui ont souligné que l'insolation et les températures élevées seraient à la base des différences morphologiques observées. Des résultats similaires sont obtenus par les travaux de Sanou et al. (2006); Hawthorne et Jonking (2006) ; Sama et Sacande (2007) sur Vitellaria paradoxa C.F Gaertn et sur P. butyraceae et Abukutsa (2010) sur les plantes indigènes africaines qui ont confirmé l'importante de la variabilité des caractères naturels de ces espèces en Afrique et qui s'explique par le climat en comparant la zone guinéocongolaise et la zone soudanienne surtout en se basant sur la largeur des feuilles et la grosseur des fruits. L'utilisation de l'espèce à des fins alimentaires, médicinales, ornementales et fourragères a influencé la structuration de la diversité de C. gynandra. Les populations aiment conserver la couleur verte dans les jardins de case à des fins utiles. Elles estiment que l'espèce à tige verte est efficace que les autres couleurs.

\section{Amélioration et sélection variétale}

Au Bénin, la population préfère plus la variété couleur verte que les autres couleurs. A cela, il faut ajouter d'autres variétés qui produisent beaucoup de feuilles et à plusieurs ramifications. Dans les marchés le mélange de toutes les variétés se vend à différentes saisons et à différents prix selon les saisons. Il est donc important de penser à la domestication de l'espèce pour être en phase avec les résultats de Djaha et Gnahoua (2014). Selon Abukutsa (2010) et Masuka et Mazarura (2012), la couleur de la tige de 
Cleome gynandra facilement visible à l'œil, est très héritable. Ainsi, l'amélioration génétique de Cleome gynandra est envisageable à partir de la variabilité mis en évidence dans cette étude et doit être orientée vers la couleur verte qui est préférée des producteurs et consommateurs et vers les caractères liés à la production de biomasse tels que la taille de la plante et le nombre de ramification. Cette amélioration pourrait se faire à partir des accessions des classes (obs $10,11,12,13,26,29,31$, et obs $5,6,12,13$, $17,18,20)$ qui présentent les meilleures performances agronomiques.

\section{Conclusion}

Cleome gynandra L. est une plante utilisée comme légume feuille et médicinale du Nord au Sud du Bénin. On la voit se développer naturellement mais aussi conservé dans les jardins de case et à l'entour des maisons. L'Analyse en composante montre trois groupes dans les trois zones climatiques $\mathrm{du}$ pays et la Classification Ascendante Hiérarchisée répartie les 31 observations en 8 classes avec des spécificités. L'espèce possède une grande variabilité en son sein. La sélection doit se poursuivre afin de mettre à la disposition des populations des variétés à haut rendement de feuilles ou biomasse et aussi la sélection doit regrouper tous les éléments caractéristiques de la plante en deux ou trois sélections.

\section{CONFLIT D'INTÉRÊTS}

Les auteurs déclarent qu'ils n'ont aucun conflit d'intérêts.

\section{CONTRIBUTIONS DES AUTEURS}

YFA a traité les données et rédigé le manuscrit; JIE a collecté les données et participé à la rédaction du manuscrit ; CAA a supervisé le travail et a lu le manuscrit; MGJD a relu le manuscrit. Tous les auteurs ont approuvé la dernière version du manuscrit.

\section{REMERCIEMENTS}

Les auteurs remercient tous les chefs de villages qui ont facilité la collecte des données et les populations locales qui ont répondues au questionnaire.

\section{REFERENCES}

Abukutsa MOO. 2010. African indigenous vegetables: strategic repositioning in the horticulture sector. Second inaugural lecture of Jomo Kenyatta University of Agriculture et Technology (JKUAT), Nairobi, Kenya, 30 avril 2010.

Agbo AE, Kouamé C, Anin AOL, Soro LC, N'zi JC, Fondio L, Gnakri D. 2014. Seasonal variation in nutritional compositions of spider plant (Cleome gynandra L.) in South Côte d'Ivoire. International Journal of Agricultural Policy and Research, 2(11): 406-413.

Akoègninou A. 2004. Recherches botaniques et écologiques sur les forêts actuelles du Bénin. Thèse d'Etat. Université de Cocody-Abidjan (Côte d'Ivoire), 326 p.

Assongba YF. 2014. Ethnobotanique, Ecologie, Répartition spatiale et statut de conservation de Dialium guineense Willd (Fabaceae) au Bénin. Journal of Applied Biosciences, 83: 7520-7534.

Bouet A, Boka A, Kouassi N. 2014. Impact de la surveillance humaine sur les dégâts d'oiseaux en riziculture pluviale. Int. J. Biol. Chem. Sci., 8(5): 2314-2319.

Dansi A, Vodouhè R, Azokpota P, Yedomonhan H, Assogba P, Adjatin A, Loko YL, Dossou-Aminon I, Akpagana K. 2012. Diversity of the neglected and underutilized crop species of importance in Benin. The Scientific World Journal, 2012 : 19 pages.

Diarrassouba N, Silué S, Fofana JI, Dago NDougba Kouamé NMT, Koffi KE. 2014. Caractérisation et évaluation des vivriers dans un système d'exploitation agricole à base de coton dans le département de Sinématiali, Côte 
d'Ivoire. Int. J. Biol. Chem. Sci., 8(4): 1656-1668.

Djaha AJB, Gnahoua GM. 2014. Contribution à l'inventaire et à la domestication des espèces alimentaires spontanées de la Côte d'Ivoire : cas des départements d'Agboville et d'Oumé. Journal of Applied Biosciences, 78 (4) : 6619-6626.

Fandohan B, Assogbadjo AE, Glèlè Kakaï R, Kyndt T, Sinsin B. 2011. Quantitative morphological descriptors confirm traditionally classified morphotypes of Tamarindus indica L. fruits. Genetic Resources and Crop Evolution, 58: 299-309.

FAOSTAT. 2010. FAO Statistics Division. FAOSTAT. http://faostat. Fao.org

Hawthorne W, Jonking C. 2006. Woody plants of western African forests. A guide to the forest trees, shrubs and lianes from Senegal to Ghana. Royal Botanic Gardens, Kew Hendeson PA, Seaby RM (2002) Community analysis package. Genet Resour Crop Evol, 58: 299-309.

Hari L. 2013. Screening phytochimique, extraction, isolation et essai de purification des flavonides des feuilles de Gynandra (Cleome) de la commune Rohero en Mairie de Bujumbura. Université du Burundi, Institut de Pédagogie Appliquée.

INSAE. 2013. Rapport provisoire du Récensement Général de la Population et de l'Habitat (RGPH), $4^{\text {ème }}$ édition. INSAE ; 182p.

IPGRI. 2002. Descriptors for Litchi (Litchi chinensis). International Plant Genetic Resources Institute, Rome, p58.

IPGRI. 2006. Descriptors for Shea tree (Vitellaria paradoxa). International Plant Genetic Resources Institute, Rome, p 63.

Keïta A. 2005. Etude de trois plantes utilisées dans le traitement traditionnel de l'ulcère gastro-duodénal dans le district de Bamako: Borassus oethiopum Mart (palmeae), Sclerocarya birrea (A. Rich). Hochst. (Anacardiaceae) et Ximenia americana L. (Olacaceae). Thése de doctorat. Bamako, p. 42.

K'Opondo FBO. 2011. Morphological characterization of selected spider plant (Cleome gynandra L.) types from western Kenya. Annals of Biological Research, 2(2): 54-64.

K'Opondo FBO, Rheenen HAV, Muasya RM. 2009. Assessment of genetic variation of selected spider plant (Cleome gynandra L.) morphotypes from western Kenya. Afr. J. of Biotechnol, 8(18): 4325-4332.

Kièbre M, Bationo Kando P, Zakaria K, Sawadogo M, Sawadogo N, Sawadogo B, Nanema RK, Traore RE. 2016. Evaluation agromorphologique d'accessions de corète potagère (Corchorus olitorius. L) du Burkina Faso. International Journal of Innovation and Applied Studies, 14(1): 198-209.

Masuka A, Goss M, Mazarura U. 2012. Morphological Characterization of Four Selected Spider Plant (Gynandra L.) Morphs from Zimbabwe and Kenya. Asian Journal of Agriculture and Rural Development, 2(4): 646 - 657.

Meda NTR, Bangou MJ, Bakassa S, MillogoRasolodimby J, Nacoulm OG. 2013. Antioxidant activity of phenolic and flavonoid fractions of Cleome gynandra and Maerua angolensis of Burkina Faso. Journal of Applied pharmaceutical Science, 3(02): 36-42.

Millogo-Rasolodimby J. 2001. L'Homme, le climat et les ressources alimentaires végétales en périodes de crise de subsistance au cours du $20^{\text {ème }}$ siècle au Burkina Faso. Thèse Doctorat, Université de Ouagadougou.

Mnzava NA, Ngwerume FC. 2004. Cleome gynandra L. Fiche de Protabase. Grubben GJH, Denton OA (Eds). PROTA (Plant Resources of Tropical Africa / Ressources végétales de l'Afrique tropicale), Wageningen, Pays Bas. 
Mnzava NA, Ngwerume FC. 2004. Cleome gynandra L. Fiche de Protabase. Grubben GJH, Denton OA (Eds). PROTA (Plant Resources of Tropical Africa / Ressources végétales de l'Afrique tropicale), Wageningen, Pays Bas.

Sama B, Sacandé M. 2007. Pentadesma butyracea Sabine, seed leaflet No. 131 December. In Millennium seed bank project, Schmidt L (ed). Forest et Landscape: Denmark.

Sanou H, Picard N, Lovett PN, Dembélé M, Korbo A, Diarisso D, Bouvet JM. 2006. Phenotypic variation of agromorphological traits of the shea tree, Vitellaria paradoxa C.F. Gaertn., in Mali. Genet Resour Crop Evol, 53: 145161.

Short PS. 2011. Capparaceae. In Flora of the Darwin Region, Short PS, Cowie ID (Eds). Northern Territory Herbarium, Department of Natural Resources, Environment, the Arts and Sport. Vol. 1, pp. 1-24.
Silué DS. 2009. Pider plant: An indigenous species with many uses. AVRDC-The World Vegetable Center Regional Center for Africa, Duluti, Arusha Tanzania: 09719.

Soro CL, Ocho-Anin AtchibrI AL, Armand KKK, Christophe K. 2012. Evaluation de la composition nutritionnelle des légumes feuilles. J. Appl. Biosci., 51: 3567-3573.

Thiombiano A, Kampmann D. 2010. Atlas de la biodiversité de l'Afrique de l'Ouest, Tome II : Burkina Faso, Ouagadougou et Frankfurt/Main.

Zakaria K, Bationo Kando P, Kiswendsida Nanema R, Sawadogo M, Zongo J-D. 2015. Evaluation agromorphologique d'accessions de corète potagère (Corchorus olitorius. L) du Burkina Faso. International Journal of Innovation and Applied Studies, 11(1): 156-166. 